\title{
The study of extraneous conditions that affect tilt-based pointer movements
}

\author{
Artūras Serackis ${ }^{1}$, Darius Miniotas ${ }^{2}$, Andrius Katkevičius ${ }^{3}$, Audrius Krukonis ${ }^{4}$, \\ Darius Plonis ${ }^{5}$ \\ 1,2,3,4,5 Vilnius Gediminas Technical University, Lithuania \\ E-mails: ${ }^{1}$ arturas.serackis@vgtu.lt (corresponding author), ${ }^{2}$ darius.miniotas@vgtu.lt, \\ ${ }^{3}$ andrius.katkevicius@vgtu.lt, ${ }^{4}$ audrius.krukonis@vgtu.lt, ${ }^{5}$ darius.plonis@vgtu.lt \\ (Received 19 September 2016; accepted 21 September 2016)
}

\begin{abstract}
Introduction: With the rapid evolution of mobile devices, there is also a tremendous growth in their applications. This triggers new research on more efficient techniques of humancomputer interaction. To point at an object of interest seen on the screen of a mobile device, various new methods were suggested recently.

Methods: This paper presents the results of a user study that employed tilting as a technique for entering text. The independent variables in the user study were mobility (sitting, walking, sitting in the moving bus) and keyboard size $(5 \times 3,10 \times 4)$. The experiment involved 50 participants aged from 22 to 65 .

Results: In the walking condition, it took on average $11.3 \%$ more time for participants to complete the task compared to the sitting condition with $5 \times 3$ keyboard, and $45.1 \%$ more time compared to the sitting condition with $10 \times 4$ keyboard. Keyboard size had a marked influence on task completion time. In addition, task completion time while traveling by bus was $3.2 \%$ longer than that observed for the walking condition with $5 \times 3$ keyboard. Surprisingly, task completion time with $10 \times 4$ keyboard while traveling by bus was $10.4 \%$ shorter compared to the walking condition. Error rate and movement efficiency were investigated additionally to find out the explanation for such performance data.
\end{abstract}

Keywords: mobile text input, tilt input, keyboard size, performance measurement.

\section{Introduction}

Research area of human-computer interaction (HCI) is becoming increasingly important because of the development of mobile technologies and the rapid growth in their usage. The task of the research is to make the usage of mobile devices user friendly by focusing on quality, effectiveness and efficiency of the graphical user interface (GUI). These objectives can be achieved in different approaches: studies of better hierarchy of information presentation [1], studies of better data input type [2].

According to [3], there are three main input facilities for mobile devices that are on the market: the keyboard, the stylus with the touch screen, and the scroll wheel. However, there are other input types such as tilt-based input, facial tracking input [4], and voice input [5]. Tilt-based input is commonly used for maintaining correct screen orientation when the device is rotated; this is also a popular input method for gaming with mobile devices [6], or it can be used for remote control in other embedded devices [7]. Exploration of possibilities for tilt-based interaction still requires research effort.

Our research focuses on the investigation of the abilities to perform practical tasks by applying the tilt-based control of the pointer. Two independent variables explored in this study were mobility (sitting, walking around a circular table, travelling in the moving bus) and keyboard size $(5 \times 3,4 \times 10)$ giving six different conditions for experimental investigation. The study is concluded with a report on the user feedback [8]. 


\section{Methods}

The various factors that can influence tilt control, separately along the three axes of wrist movement showed that users could control comfortably at least 16 levels on the pronation/supination axis [9]. Tilt-based input enhances the user experience when compared to key-based input [10].

Participants. All participants were from Vilnius, the capital of Lithuania, and aged between 22 and 65 years. The total number of participants was equal to 50. Anybody who expressed willingness to participate in the experiment was allowed to do that with no restrictions. It was only required that at least two participants were from the following four age groups: 20-30 years old (the youngest), 31-45 years old (younger middle-aged), 46-60 years old (older middle-aged), and 61-80 old (the oldest). Participants were categorized to four groups according to [11]. Participants from different age groups were chosen because user age is an important criterion and people adapt differently to new user interfaces [12]. All participants were unpaid volunteers in the study. Experience of participants in working with mobile devices was taken into account. All the participants had been trained on how to work with the tilt-input before the study began.

The experimental interface. In this study, the tilt of the device was directly mapped to the position of the ball. This allowed quick and easy control of the ball's position. The zero position of the interface (corresponding to the ball is in the centre of the screen) occurs when the device is parallel to the ground in both the pitch and roll axes (Fig. 1). To avoid an unintended start, each task commenced with the ball resting in one corner of the screen. The application controlled the sequence of the tasks presented to the participants. They had to select 10 targets in turn from either a $5 \times 3$ or $10 \times 4$ grid. To complete the selection, the participant was to move the ball to the target square and dwell upon it for a period of $500 \mathrm{~ms}$. A target was approximately $3 \mathrm{~cm} \times 2.5 \mathrm{~cm}$ in size to resemble a real character. The application selected the targets at random; the target was identified by highlighting it. The highlight became more intense when the ball appeared in the target's area. A beep could be heard after each successful selection.

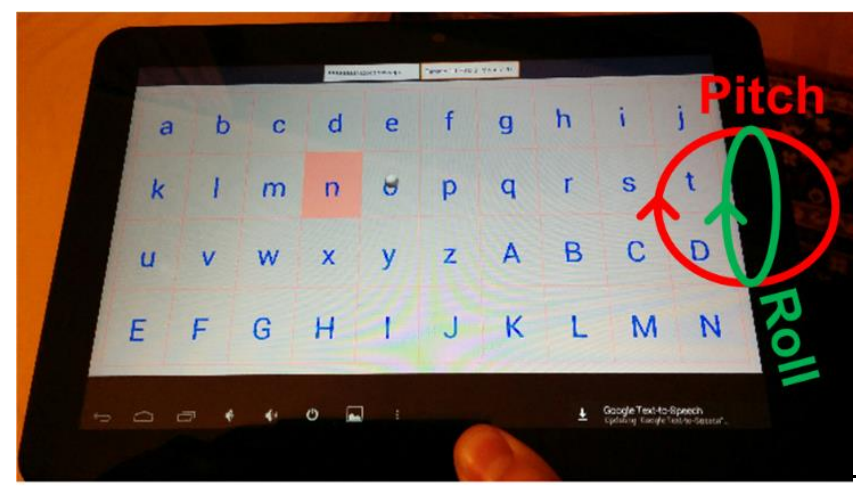

Fig. 1. Participant completing tilt input task (pitch and roll axis indicated)

The application allowed all "characters" on the interface to be selected throughout the task, with erroneous selections recorded. After each successful selection, the corresponding character appeared at the top of the screen. The number of remaining targets was displayed on the interface along with the task condition.

Procedure. For each participant the brief demonstration and key information about the task was provided. Participants were then given a device each and allowed to complete a practice task sitting on the chair and selecting 10 targets. The data from practice phase was recorded but was 
not used in this paper. After the practice task, the participants completed six tasks (each task was fulfilled 5 times with 10 inputs per task) in each condition before moving on to the next one.

The study was performed with each participant individually with the changing sequence of conditions. Some participants fulfilled the task sitting on the chair first, some walking around the table and some participants started from fulfilling the task sitting in the bus. Task execution order was changing for every participant in order to get adequate results because participant gets some experience after each fulfilled task. Participants fulfil the task 5 times in every condition (sitting, walking, and travelling in bus) with two different keyboard sizes $(5 \times 3$ or $10 \times 4)$. Over all every participant fulfils the task 30 times.

Walking path was around a circular table. Participants were asked to walk at normal speed, at which they usually walk. It is difficult to maintain the same conditions while traveling in bus for experiments but in order to maintain conditions as uniform as possible the same bus route was used during peak hours from 5 till $6 \mathrm{pm}$.

Each participant used an identical Android device Exynos4412 Prime Quad Development Platform ODROID-Q2. In the sitting condition, participants used chairs. The study was designed to last no longer than 20 minutes per participant (the time required to wait for the proper bus was not included) to ensure that the participant did not tire or lose interest.

\section{Results}

Three simplest performance metrics were used: task completion time, the error rate and the efficiency of ball movement. Error rate in this context is the ratio of inadvertent, or extra, selections to correct selections. As there were always 10 correct selections per task, two extra inadvertent selections would result in an error rate of $2 / 10=20 \%$. Efficiency of ball movement is the ratio of the minimum distance between targets and the actual distance moved by the ball. $100 \%$ efficiency implies a straight-line movement from the centre of each target to the next target.

From the data collected, the shortest task completion time was obtained while sitting. Task completion time was $11.1 \%$ longer while walking with a $5 \times 3$ keyboard size and $14.5 \%$ longer while walking with the $10 \times 4$ keyboard size (Fig. 2). Unexpectedly, the task completion time decreased while travelling in the bus comparing with task completion time while walking and using the 10x4 keyboard size.

The smallest error rate was while walking (Fig. 3). It was equal to $51.6 \%$ with $5 \times 3$ keyboard size and $51.6 \%$ with $5 \times 3$ keyboard size. The error rate was bigger while travelling in the bus and unexpectedly the biggest error rate was while sitting comparing with walking and travelling in the bus.

The biggest distance efficiency was received while sitting and the smallest efficiency while walking with both $5 \times 3$ and $10 \times 4$ keyboard sizes (Fig. 4). These results correlate with estimated task completion times and error rates. If the time of task completion is less it gives with the smaller error rate and higher efficiency.

The investigation also showed that age of participants has influence to the task completion time, error rate and distance efficiency.

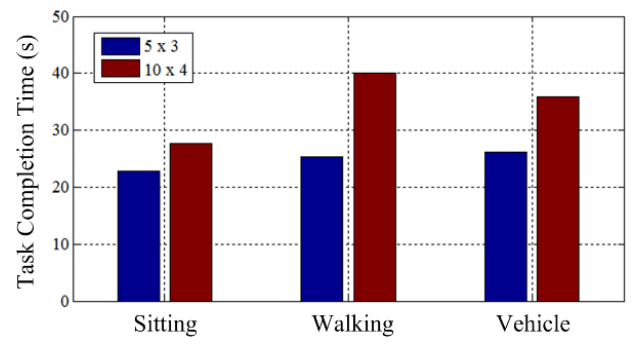

Fig. 2. The participant performs the task while walking around a circular table 


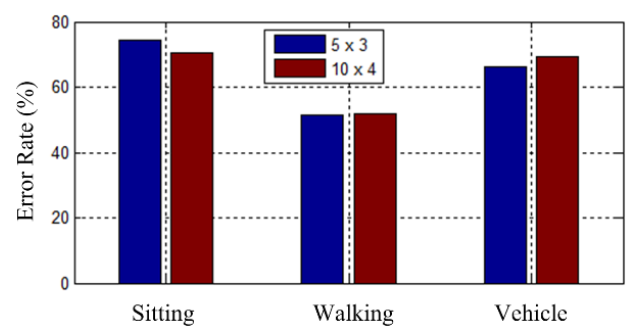

Fig. 3. Mean error rates for all conditions

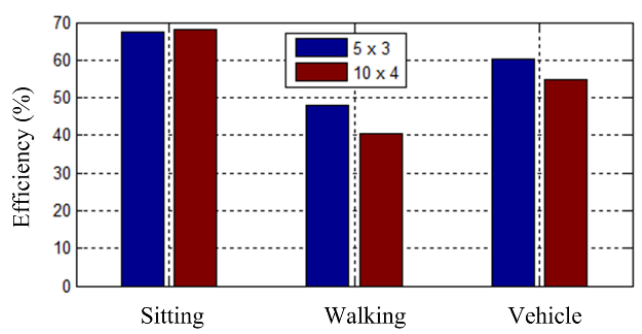

Fig. 4. Mean efficiency rates for all conditions

The older middle-aged and oldest participants had bigger task completion time and error rates. In respect of each participant individually youngest and younger middle-aged participants adapted to conditions of the game faster than others.

Unexpectedly the task completion time while travelling in bus almost unchanged and increased $3.2 \%$ (an additional $\sim 0.8$ seconds) with $5 \times 3$ keyboard size and decreased $10.4 \%$ (an additional $\sim 4.18$ seconds) with $10 \times 4$ keyboard size compared to walking. Participants had less distraction and had better control over the movement of the device while sitting in the bus compared with walking.

The movement of the body is partly transmitted to the device to make accurate tilt-input more challenging while walking. The attention of the participants also was triggered by the additional navigation tasks around the course and turn in the corners. The quality of the results could be influenced by factor that participants more concentrate on more complex task. Therefore, lower error rate is obtained while walking or travelling in the bus while comparing with sitting.

\section{Conclusions}

As demonstrated by this experiment, participants from all the four age groups investigated were able to successfully select targets on mobile devices using the text entry method described here. Investigation under the six different conditions revealed that disturbances of the real settings such as shaking while sitting on a bus do not significantly affect user performance. The age of participants influences the task completion time, error rate, and efficiency. The oldest participants had a longer task completion time and a higher error rate. The youngest participants were able to adapt to the conditions of the game faster than the other groups. The performance data could be influenced by the factor that participants tended to concentrate more on a more complex task.

\section{Acknowledgements}

We would like to thank Scott MacKenzie from York University in Canada for his valuable comments, insights, and sharing his experimental software with us. This research was funded by a grant (No. MIP-083/2015) from the Research Council of Lithuania. 


\section{References}

[1] Hao, J., et al. 2010. Visualizing and navigating hierarchical information on mobile user interfaces, International Journal of Advanced Intelligence 2(1): 81-103.

[2] Huang, K. Y. 2009. Challenges in human-computer interaction design for mobile devices, in Proceedings of the World Congress on Engineering and Computer Science, October 20-22, 2009, San Francisco, USA. Hong Kong: Newswood Limited, 20-22.

[3] Muhanna, A. 2007. Exploration of human-computer interaction challenges in designing software for mobile devices. Master's thesis. Reno: University of Nevada. $104 \mathrm{p}$.

[4] Newman, K. E.; Hobbs, E.; Blei, M. 2013. A tablet based hands free interface to nurse communication in the standard hospital room for limited mobility patients, Information Engineering Letters 3(3): 5056.

[5] Hwang, Y. et al. 2012. Developing a voice user interface with improved usability for people with dysarthria, in Proceedings of 13th International Conference ICCHP2012, July 11-13, 2012, Linz, Austria. Berlin Heidelberg: Springer-Verlag, 117-124.

http://dx.doi.org/10.1007/978-3-642-31534-3_18

[6] Marzo, A.; Bossavit, B.; Hachet, M. 2014. Evaluating controls for a point and shoot mobile game: augmented reality, tilt and touch, in IEEE International Symposium on ISMAR-MASH'D, September 10-12, 2014, Munich, Germany. Piscataway: IEEE, 59-62. http://dx.doi.org/10.1109/ISMAR-AMH.2014.6935439

[7] Boring, S.; Jurmu, M.; Butz, A. 2009. Scroll, tilt or move it: using mobile phones to continuously control pointers on large public screens, in Proceedings of the 21 st Annual Conference of the Australian Computer-Human Interaction Special Interest Group (CHISIG), November 23-27, 2009, Melbourne, Australia. New York: ACM, 161-168. http://dx.doi.org/10.1145/1738826.1738853

[8] MacKenzie, I. S.; Teather, R. J. 2012. FittsTilt: the application of fitts' law to tilt-based interaction, in Proceedings of the 7th Nordic Conference on Human-Computer Interaction NordiCHI 2012. October 14-17, 2012, Copenhagen, Denmark. New York: ACM, 568-577. http://dx.doi.org/10.1145/2399016.2399103

[9] Partridge, K., et al. 2002. TiltType: accelerometer-supported text entry for very small devices, in Proceedings of the ACM Symposium on User Interface Software and Technology UIST2002, October 27-30, 2002, Paris, France. New York: ACM, 201-204. http://dx.doi.org/10.1145/571985.572013

[10] Gilbertson, P.; Coulton, P.; Chehimi, F. 2008. Using "tilt" as an interface to control "no-button" 3-D mobile games, Computers in Entertainment, 6(3): 1-13. http://dx.doi.org/10.1145/1394021.1394031

[11] Wilkowska, W.; Gaul, S.; Ziefle, M. 2010. A small significant difference - the role of gender on acceptance of medical assistive technologies, in Proceedings of 6th Symposium of Human-Computer interaction and Usability Engineering USAB2010, November 4-5, 2010, Klagenfurt, Austria. Berlin Heidelberg: Springer, 82-100. http://dx.doi.org/10.1007/978-3-642-16607-5_6

[12] Arab, F.; Malik, Y.; Abdulrazak, B. 2013. Evaluation of PhonAge: an adapted smartphone interface for elderly people, in Proceedings of 14th IFIP TC 13 International Conference, September 2-6, 2013, Cape Town, South Africa. Berlin Heidelberg: Springer, 547-554.

http://dx.doi.org/10.1007/978-3-642-40498-6_44 